\title{
How do 66 European institutional review boards approve one protocol for an international prospective observational study on traumatic brain injury? Experiences from the CENTER-TBI study
}

Marjolein Timmers $^{1 \dagger}$, Jeroen T. J. M. van Dijck ${ }^{2 \dagger}$, Roel P. J. van Wijk ${ }^{2}$, Valerie Legrand ${ }^{3}$, Ernest van Veen ${ }^{1,4}$, Andrew I. R. Maas ${ }^{5,6}$, David K. Menon ${ }^{7}$, Giuseppe Citerio ${ }^{8,9}$, Nino Stocchetti ${ }^{10,11}$, Erwin J. O. Kompanje ${ }^{1,12^{*}}$ (D) and The CENTER-TBI investigators and participants

\begin{abstract}
Background: The European Union (EU) aims to optimize patient protection and efficiency of health-care research by harmonizing procedures across Member States. Nonetheless, further improvements are required to increase multicenter research efficiency. We investigated IRB procedures in a large prospective European multicenter study on traumatic brain injury (TBI), aiming to inform and stimulate initiatives to improve efficiency.

Methods: We reviewed relevant documents regarding IRB submission and IRB approval from European neurotrauma centers participating in the Collaborative European NeuroTrauma Effectiveness Research in Traumatic Brain Injury (CENTER-TBI). Documents included detailed information on IRB procedures and the duration from IRB submission until approval(s). They were translated and analyzed to determine the level of harmonization of IRB procedures within Europe.
\end{abstract}

Results: From 18 countries, 66 centers provided the requested documents. The primary IRB review was conducted centrally $(N=11,61 \%)$ or locally $(N=7,39 \%)$ and primary IRB approval was obtained after one $(N=8,44 \%)$, two $(N=6,33 \%)$ or three $(N=4,23 \%)$ review rounds with a median duration of respectively 50 and 98 days until primary IRB approval. Additional IRB approval was required in 55\% of countries and could increase duration to 535 days. Total duration from submission until required IRB approval was obtained was 114 days (IQR 75-224) and appeared to be shorter after submission to local IRBs compared to central IRBs (50 vs. 138 days, $p=0.0074$ ).

(Continued on next page)

* Correspondence: erwinkompanje@me.com

${ }^{\dagger}$ Marjolein Timmers and Jeroen T. J. M. van Dijck contributed equally to this

work.

'Department of Intensive Care, Erasmus MC - University Medical Centre

Rotterdam, P.O. Box 2040, 3000 CA Rotterdam, the Netherlands

${ }^{12}$ Department of Medical Ethics and Philosophy of Medicine, Erasmus MC -

University Medical Center Rotterdam, Rotterdam, the Netherlands

Full list of author information is available at the end of the article

C C The Author(s). 2020 Open Access This article is licensed under a Creative Commons Attribution 4.0 International License, which permits use, sharing, adaptation, distribution and reproduction in any medium or format, as long as you give appropriate credit to the original author(s) and the source, provide a link to the Creative Commons licence, and indicate if changes were made. The images or other third party material in this article are included in the article's Creative Commons licence, unless indicated otherwise in a credit line to the material. If material is not included in the article's Creative Commons licence and your intended use is not permitted by statutory regulation or exceeds the permitted use, you will need to obtain permission directly from the copyright holder. To view a copy of this licence, visit http://creativecommons.org/licenses/by/4.0/. The Creative Commons Public Domain Dedication waiver (http://creativecommons.org/publicdomain/zero/1.0/) applies to the data made available in this article, unless otherwise stated in a credit line to the data. 
(Continued from previous page)

Conclusion: We found variation in IRB procedures between and within European countries. There were differences in submission and approval requirements, number of review rounds and total duration. Research collaborations could benefit from the implementation of more uniform legislation and regulation while acknowledging local cultural habits and moral values between countries.

Keywords: Research ethic committees, European Union, Health-care research, CENTER-TBI, Harmonization

\section{Background}

A Research Ethics Committee or Institutional Review Board (collectively referred to as IRB in the remainder of this manuscript) is appointed to review research protocols to ensure their compliance with ethical standards and national laws. IRBs have an essential role in (clinical) research to protect the dignity, fundamental rights, safety, and well-being of research participants and their formal approval is compulsory before a clinical study can start [1]. Although several international models exist to improve the harmonization of ethical principles, the functioning of IRBs are subject to national legislation and regulation, which refine their structure and function to better serve local needs and cultural preferences [2, 3]. Approval of research protocols submitted to IRBs is subject to these differences, which may complicate the conduct of international research.

Managing variations in IRB procedures is important because of the increasing number of research initiatives which involve multiple European Union (EU) Member States [4-6]. Variation could be improved by harmonization of European law, which is the process of creating uniformity in laws, regulations and practices between countries. Regarding research and IRB procedures, lack of procedural harmonization 'leads to a complex and uncertain framework for ethical review and for participant information consent, resulting in numerous inefficiencies in observational studies' [7]. Greater procedural harmonization is generally considered desirable, because it could improve quality and efficiency of healthcare research by decreasing costs, increasing statistical validity, [8-10] optimizing data management, [10] allowing choice of relevant and generalizable outcome variables, [9] promoting uniform product safety regulations [8] and minimizing waste of resources due to inefficiencies [8].

Although most IRBs have websites that describe the local submission process and provide access to submission guidelines and forms, up to date systematic information on IRB procedures and their level of harmonization in European health-care research is scarce. We are aware of only one previous meta-analysis on IRB procedures across European countries from 2005 to 2007 that was also related to research involving acutely mentally incapacitated individuals [6]. The
Collaborative European Neurotrauma Effectiveness Research in Traumatic Brain Injury (CENTER-TBI) study is a large observational study conducted in many countries across Europe that provides a unique opportunity to assess European IRB policies and procedures [11].

This study aims to improve the efficiency of future research initiatives by quantifying the differences in IRB procedures through analyzing the procedural details, problems and challenges that researchers encountered in obtaining IRB approval for the general research protocol of the CENTER-TBI study.

\section{Methods \\ Study setting}

The Collaborative European NeuroTrauma Effectiveness Research in Traumatic Brain Injury (CENTER-TBI, www.center-tbi.eu) Core study is a prospective observational study on traumatic brain injury (TBI), which was conducted between December 2014 and December 2017 in 63 neurotrauma centers across Europe and Israel [11, 12]. The study included patients with TBI of all severities, and aims to improve characterization of TBI, in order to facilitate the development of precision medicine approaches and to identify best practices by using a comparative effectiveness research (CER) approach [11-14]. In the context of the project highquality Personal Health related Data (PHD) were collected with repositories for neuro-imaging, DNA, and serum biomarkers. Prior to the study start and collection of clinical data, a uniform CENTER-TBI research protocol including all relevant documents was sent to all responsible IRBs to ensure its legal, ethical and statistical soundness and to obtain IRB approval.

A total of 68 centers from 19 countries initially submitted applications for IRB approval. Because this article focuses on IRB approval in Europe, two centers from Israel were excluded from our analysis. The 66 center that participated in this present study are from Austria $(N=2)$, Belgium $(N=5)$, Denmark $(N=2)$, Finland $(N=$ $2)$, France $(N=7)$, Germany $(N=4)$, Hungary $(N=3)$, Italy $(N=8)$, Latvia $(N=3)$, Lithuania $(N=2)$, the Netherlands $(N=7)$, Norway $(N=3)$, Romania $(N=1)$, Serbia $(N=1), \quad$ Spain $\quad(N=4), \quad$ Sweden $\quad(N=2)$, Switzerland $(N=1)$, and the United Kingdom (UK), 
$(N=9)$. Sixty-one European centers were initiated and actively enrolled patients in the study.

\section{Data collection and administration}

All IRB submission documents, communication records and approval documents were collated per center by the Contract Research Organization, ICON plc (ICON), directly after final approval of IRBs [15]. ICON is a global company operating in the healthcare industry that was responsible for the clinical monitoring of CENTER-TBI data. The received IRB documents were obtained in 15 different languages (Danish, Dutch, English, Finnish, French, German, Hungarian, Italian, Latvian, Lithuanian, Norwegian, Romanian, Serbian, Spanish, and Swedish) and were partly translated before analysis. The authors contacted the principle investigators to obtain additional information to minimize the amount of unclear or missing data. Identifiable information was deleted to protect the privacy of stakeholders. This resulted in a final set of documents, that was analyzed for this study.

\section{Analyses}

We assessed the IRB review procedures by using the final set of documents and aimed to answer the following research questions in order to evaluate differences in obtaining IRB approval (1) Was the study considered to be observational or interventional? (2) Was the research protocol to be submitted to a central IRB or local IRB for primary IRB review and primary IRB approval? (3) Was additional IRB review required after primary IRB approval had already been obtained? If yes, to what extent? (4) How many review rounds were conducted before primary IRB approval was obtained? What were the reasons? (5) What was the time between protocol submission and obtaining the required IRB approval to start the study? The use of 'primary' in this context should be interpreted as first in an order and 'additional' as second in an order, without including a statement on importance.

To elaborate on the fifth question, we reconstructed six timeframes regarding the primary IRB review procedure: (1) time between protocol submission and primary IRB approval or first IRB reaction, (2) time between first IRB reaction and first reaction of researcher, (3) time between first reaction of researcher and primary IRB approval or second IRB reaction, (4) time between second IRB reaction and second reaction researcher, (5) time between second reaction researcher and primary IRB approval, and (6) total time between protocol submission and primary IRB approval. The existence of these timeframes naturally depended on the actual procedure. Data on any additional IRB review focused only on the duration of this particular review until the required IRB approval was obtained.
In order to assess regional variation, countries were grouped into six regions based on the United Nation geo-scheme: Baltic States (Latvia, and Lithuania), Eastern Europe (Hungary, Romania, and Serbia), Northern Europe (Denmark, Finland, Norway, and Sweden), Southern Europe (Italy, and Spain), the United Kingdom (UK), and Western Europe (Austria, Belgium, France, Germany, the Netherlands, Switzerland) [16]. Incomplete data was marked 'Missing' (M) and all timeframes were reported in days.

To determine significant differences between the time from submission till approval of the research protocol between primary local IRBs and primary central IRBs, we performed a Mann-Whitney $U$ test (continuous). Analyses were performed using R version 3.6.0. Finally, a descriptive analysis of questions, comments and answers from both IRB and researcher during the IRB review procedure was performed to summarize the problems and challenges that researchers encountered in obtaining IRB approval. IRB reactions were categorized and reported by their appearance: (1) Procedure, (2) Blood collection and biomarkers, (3) MRI, (4) Privacy and data security, (5) Other.

\section{Results}

A total of 66 neurotrauma centers from 18 countries were included in this analysis. Most centers were located in Western Europe $(N=26,39 \%)$ and least in Eastern Europe $(N=5,8 \%)$ and the Baltic States $(N=5,8 \%)$. Most participating centers were from the UK $(N=9)$, followed by Italy $(N=8)$, The Netherlands and France $(N=7)$ (Table 1). In all countries the local principal investigators were responsible to submit the general CENTER-TBI research protocol for IRB review and IRB approval.

\section{Observational or interventional}

The majority of countries $(N=14,78 \%)$ considered the study to be observational, while others judged it to be observational with diagnostic interventions (The Netherlands), interventional (France, Hungary) and observational and interventional (Serbia) (Table 1).

\section{Primary central or primary local IRB review}

Primary IRB review started directly after protocol submission and was considered 'central' when submitted to a central institution or an institution that was part of a national network $(N=11,61 \%)$. There were three options: (1) Primary central IRB approval had a national impact and applied to all participating centers within a country, without the need for additional IRB review ( $N=5$; Denmark, Finland, France, Norway, Sweden). (2) Primary central IRB approval only allowed study start in the research centers associated with the approving IRB. 
Table 1 Baseline study information

\begin{tabular}{|c|c|c|c|}
\hline $\begin{array}{l}\text { Region } \\
\text { Country }\end{array}$ & $\begin{array}{l}\text { Centers } \\
(\mathrm{N})\end{array}$ & $\begin{array}{l}\text { Central or local } \\
\text { IRB review }\end{array}$ & $\begin{array}{l}\text { IRB decision on study } \\
\text { type }\end{array}$ \\
\hline Baltic States & 5 & & \\
\hline Latvia & 3 & Local $^{a}$ & Observational \\
\hline Lithuania & 2 & Local & Observational \\
\hline Eastern Europe & 5 & & \\
\hline Hungary & 3 & Central & Interventional \\
\hline Romania & 1 & Local & Observational \\
\hline Serbia & 1 & Local & $\begin{array}{l}\text { Observational and } \\
\text { Interventional }\end{array}$ \\
\hline Northern Europe & 9 & & \\
\hline Denmark & 2 & Central & Observational \\
\hline Finland & 2 & Central & Observational \\
\hline Norway & 3 & Central & Observational \\
\hline Sweden & 2 & Central & Observational \\
\hline Southern Europe & 12 & & \\
\hline Italy & 8 & Central & Observational \\
\hline Spain & 4 & Local & Observational \\
\hline United Kingdom & 9 & & \\
\hline United Kingdom & 9 & Central $^{b}$ & Observational \\
\hline Western Europe & 26 & & \\
\hline Austria & 2 & Local & Observational \\
\hline Belgium & 5 & Central & Observational \\
\hline France & 7 & Central & Interventional \\
\hline Germany & 4 & Central & Observational \\
\hline Netherlands & 7 & Central & $\begin{array}{l}\text { Observational with } \\
\text { diagnostic interventions }\end{array}$ \\
\hline Switzerland & 1 & Local & Observational \\
\hline
\end{tabular}

Other participating centers in the country required approval after an additional extensive local IRB review. This involved the re-evaluation of the entire protocol and applicable ethics $(N=4 ;$ Belgium, Germany, Hungary, Italy). (3) Primary central IRB approval only allowed study start in the research centers associated with the approving IRB. Other participating centers required additional approval after marginal local IRB review, mainly assessing local feasibility $(N=2$; UK, The Netherlands) (Fig. 1).

Primary IRB review was considered 'local' when the protocol was submitted to an independent 'local' IRB. Obtained primary local IRB approvals only applied to the associated research centers and allowed study start without any additional requirements $(N=7$; Austria,
Switzerland, Spain, Lithuania, Latvia, Romania, Serbia). Primary local IRB review could be performed simultaneously in each independent IRB (Fig. 1).

For every protocol submission, there were two outcome options after IRB review: (1) the required (primary or additional) IRB approval had been obtained and the study could start, or (2) researchers were asked to answer questions or make protocol changes, which was followed by an extra IRB review round. This process varied between IRBs and was repeated until the required IRB approval was eventually obtained. None of the submissions in this study were rejected.

\section{IRB review rounds}

Eight countries (44\%), including all countries from Eastern Europe and the Baltic State, obtained primary IRB approval in the first round after submission, while six countries (Austria, Belgium, France, Finland, Spain and $\mathrm{UK}$ ) required one extra review round and four countries (Denmark, Germany, Norway and Sweden) required two extra review rounds (Fig. 2). Extra review rounds were found in $73 \%$ of centers after primary central IRB submission and in $20 \%$ after primary local IRB submission.

Several IRBs commented on different aspects of the protocol: selection criteria $(n=3,38 \%)$, patient/proxy consent $(n=4,50 \%)$, and information forms $(n=3,38 \%)$. Also, specific questions were asked on possible nonstandard care factors in particular MRI scans $(N=4)$, blood sample collection $(N=4)$. Four questions were asked about privacy and data security, mainly related to the period after study completion. All relevant information can be found in the supplementary files.

\section{Duration from protocol submission to IRB approval}

The median time from protocol submission until the required IRB approval was obtained to start the study was 114 days (IQR 75-224). The fastest required IRB approval was obtained after one day in Serbia and Romania, whereas the longest time was found in a center in the UK ( 535 days). Obtaining central IRB approval (138 days, IQR: $91-229)$ took significantly longer $(p=$ 0.0074 ) than obtaining local IRB approval (50 days, IQR: 29-102) (Table 2).

In Norway and Denmark, the majority of time from submission to primary central IRB approval was spent by researchers (67 and 69\%, respectively), while in France $(95 \%)$ and Hungary $(71 \%)$ most time was consumed by IRBs. Regarding primary local IRB submissions, researchers only accounted for $12 \%$ of time in Spain and 21\% in Austria (Fig. 2).

Additional IRB review rounds after primary central IRB review were required in 55\% of countries. An additional marginal (feasibility) review had a median 

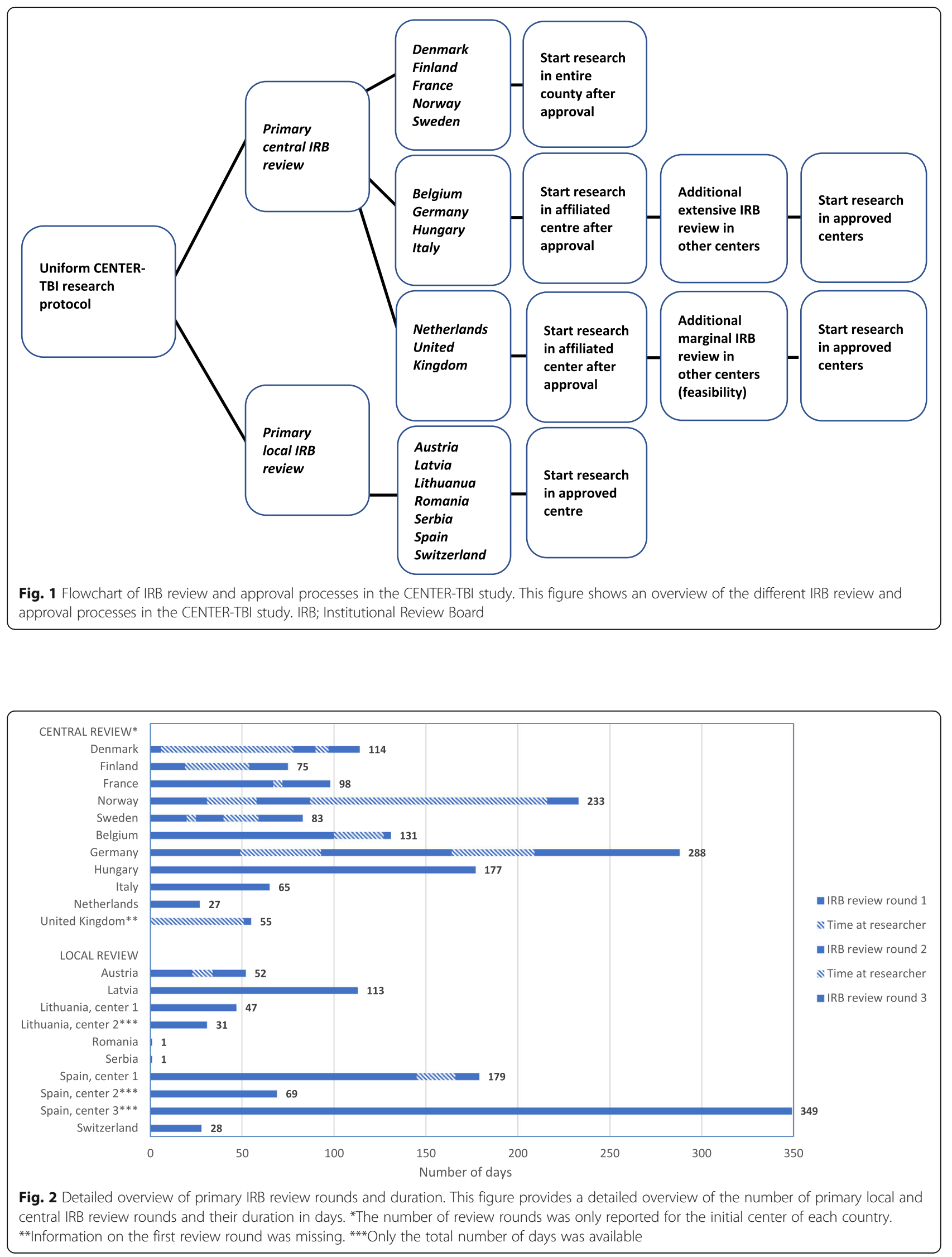
Table 2 Duration of protocol submission until required IRB approval before study start

\begin{tabular}{llll}
\hline & Duration (days) & Centers (N) & Missing (N) \\
\hline All centers & $114(75-224)$ & 58 & 8 \\
Local review & $50(29-102)$ & 10 & 4 \\
Central review & $138(91-229)^{\mathrm{b}}$ & 48 & 4 \\
- Central (1) & $98(94-114)$ & 16 & 0 \\
- Central (2) & $189(140-270)$ & 17 & 3 \\
- Central (3) & $104(62-224)$ & 15 & 1
\end{tabular}

Local review: Obtained primary local IRB approvals only applied to the associated research centers and allowed study start without any additional requirements Central (1): Primary central IRB approval with national impact, applying to all center within a country, without the need for additional local IRB review Central (2): Primary central IRB approval only allowed study start in the research centers associated with the approving IRB. Other participating centers required approval after additional extensive local IRB review Central (3): Primary central IRB approval only allowed study start in the research centers associated with the approving IRB. Other participating centers required approval after additional marginal local IRB review ${ }^{\mathrm{a}}$ Duration was reported in median number of days (IQR)

${ }^{\mathrm{b}}$ Group differences between local and central review were significant $(\mathrm{P}=$ 0.0074, Mann-Whitney U) duration of 104 days (IQR: 62-224), whereas an additional extensive IRB review took 189 days (IQR: 140 270) (Table 3).

Variation between centers within countries was least in Lithuania (31 to 47 days), Germany (288 to 312 days), Belgium (131 to 155 days), and Hungary (177 to 204 days), compared to Spain (69 to 349 days), the Netherlands (27 to 224 days), the UK (58 to 535 days), and Italy (65 to 288 days) (Table 3 ).

\section{Discussion}

This study shows variation in IRB procedures between and within European countries, indicating a lack of uniform legislation and regulation, or inconsistencies in how such legislation or regulation were implemented. In some countries, a primary central IRB approval was sufficient for study initiation, while others required an additional IRB review at the participating site. Also, the number of review rounds, duration until IRB approval, and the nature of questions and comments from the

Table 3 Duration from submission to required IRB approval before study start per country and study center

\begin{tabular}{|c|c|c|c|c|c|c|c|c|c|c|}
\hline \multirow[t]{3}{*}{ Country } & \multirow{3}{*}{$\begin{array}{l}\text { Central or } \\
\text { local IRB } \\
\text { review }\end{array}$} & \multicolumn{9}{|c|}{ Duration in days } \\
\hline & & \multicolumn{9}{|c|}{ Centre } \\
\hline & & 1 & 2 & 3 & 4 & 5 & 6 & 7 & 8 & 9 \\
\hline Denmark & Central (1) & 114 & 114 & & & & & & & \\
\hline Finland & Central (1) & 75 & 75 & & & & & & & \\
\hline France & Central (1) & 98 & 98 & 98 & 98 & 98 & 98 & 98 & & \\
\hline Norway & Central (1) & 233 & 233 & 233 & & & & & & \\
\hline Sweden & Central (1) & 83 & 83 & & & & & & & \\
\hline Belgium & Central (2) & 131 & 138 & 141 & 257 & M & & & & \\
\hline Germany & Central (2) & 288 & 296 & 312 & M & & & & & \\
\hline Hungary & Central (2) & 177 & 200 & 204 & & & & & & \\
\hline Italy & Central (2) & 65 & 70 & 139 & 141 & 155 & 261 & 273 & 288 & \\
\hline Netherlands & Central (3) & 27 & 46 & 91 & 209 & 223 & 224 & M & & \\
\hline United Kingdom ${ }^{a}$ & Central (3) & 58 & 61 & 63 & 84 & 104 & 157 & 229 & 282 & 535 \\
\hline Austria & Local & 52 & M & & & & & & & \\
\hline Latvia & Local & 113 & M & M & & & & & & \\
\hline Lithuania & Local & 31 & 47 & & & & & & & \\
\hline Romania & Local & 1 & & & & & & & & \\
\hline Serbia & Local & 1 & & & & & & & & \\
\hline Spain & Local & 69 & 179 & 349 & M & & & & & \\
\hline Switzerland & Local & 28 & & & & & & & & \\
\hline
\end{tabular}

Central (1): Primary central IRB approval with national impact, applying to all center within a country, without the need for additional local IRB review to start study

Central (2): Primary central IRB approval only allowed study start in the research centers associated with the approving IRB. Other participating centers required approval after additional extensive local IRB review to start study

Central (3): Primary central IRB approval only allowed study start in the research centers associated with the approving IRB. Other participating centers required approval after additional marginal local IRB review to start study

Local review: Obtained primary local IRB approvals only applied to the associated research centers and allowed study start without any additional requirements $M=$ Missing

${ }^{a}$ In the UK, the research protocol had to be submitted to an external national committee not associated to the submitting center. After primary approval by this national committee, all centers required additional IRB approval 
IRBs varied. Not all IRBs considered the study to be observational, demonstrating a different way of understanding the study. The apparent lack of integration and harmonization in this context suggests that the efficiency of European research collaborations could benefit from improving knowledge on the existing variation in procedures, inefficiencies and differences in value systems between and within countries.

The duration from protocol submission to required IRB approval was highly variable and ranged from one day up to nearly one year. In literature, differences between IRB procedures were also reported and IRB review durations varied from weeks to several months $[6,17]$. The difference in total duration between primary central and primary local IRB approval could respectively be overestimated and underestimated by the short primary IRB review times in Serbia and Romania and the missing data of the first review round for the UK. The difference is not necessarily related to the number of review rounds, but might be more explained by the reason and nature (primary central/local review or extensive/marginal additional local review) of the extra review round(s), the accompanying amount of work and the working speed of both IRB and research team. The influence of the latter was substantiated by our data as responding to questions from the IRB seemed to account for an important part of time in several countries (e.g. Denmark and Norway), while the majority of time in other countries (e.g. Belgium, Spain and France) was accounted for by the time taken in primary evaluation by IRBs. The exact reasons for these 'delays' could however not be derived from our data and deserves further study. They might be caused by the difficulty of requirements or questions, although, according to the communication records, IRBs mainly requested extra explanation of research procedures. Based on the IRB information requests in this study, special attention should be given to the description of inclusion criteria, informed consent procedures, patient information forms, non-standard care procedures, privacy and data security. A quick response by investigators and agreeing on a maximal turnover time of 1 month to 2 months for IRBs could already minimize substantial delay. This is also in correspondence with literature, where IRB turnover time targets range from 30 to 60 days $[17,18]$.

The question whether CENTER-TBI was an observational or an interventional study did not appear to be a clear explanation for differences in number and duration of review rounds. Interventional studies are generally subject to a more extensive review process, where observational study reviews may be more marginal. Nonetheless, duration was short in France and long in the UK. CENTER-TBI is registered as an observational study, in which 'the investigator is not acting upon study participants, but instead observing natural relationships between factors and outcomes' [19]. Two IRBs considered the study to be purely interventional. Interventional studies are studies 'where the researcher intercedes as part of the study design' [19]. An explanation for this opposing classification is that the IRBs did and did not consider the following procedures to be standard-ofcare: (1) Different amounts of additional blood draws at presentation and follow-up. (2) Neuropsychological assessments and outcome questionnaires up to a 24month follow-up. (3) Additional MRIs at sites participating in the MRI sub-study.

Extra work without clear benefits delays projects and should be avoided when possible. An additional IRB review after primary central IRB approval is usually double work and could result in an extra delay of weeks to more than a year, without always having clear benefits over the already obtained primary approval [17]. Cancelling potentially unnecessary (extensive) additional IRB review procedures could not only reduce turnover time, but also reduce costs. The exact costs of European IRB review procedures are unfortunately unknown, but the direct costs of an IRB review and approval in the US have been calculated to be $\$ 107.544$ ( $\$ 82.610$ in IRB fees and $\$ 24.934$ in labor) [20].

Delays in obtaining IRB approval not only adversely affect study initiation, but are also associated with several other risks. Long procedures with many feedback rounds will delay study start, frustrate researchers and might even endanger meeting subsidiary demands. Researchers might attempt to speed up the process by changing the protocol or submitting the protocol to IRBs that are considered to be less strict but able to process the submission the quickest. This does not necessarily serve primary research objectives and might even hamper quality and generalizability of study results.

Optimization of IRB review procedures is urgently needed as multinational collaborations in healthcare research are increasing and even promoted by multiple European research grant $[4,5,21]$. Harmonization and adequate implementation of regulatory and ethical standards between European countries could improve the present situation [7, 22]. The EU already aims to freely cooperate across borders by defining common standards and removing legal obstacles, but true harmonization of Member State laws in a research context has clearly not been established yet [21-24]. For example, the General Data Protection Regulation (GDPR) aimed to ensure a fair and transparent processing of personal data and aimed to improve patients' control over their own data [25]. The implementation and use of the GDPR however showed the difficulty of harmonization in the protection of the EU citizens in this context. This was 
especially caused by the possibility for European countries to use their own national legislation in addition to the GDPR, which does not improve the desired harmonization.

Harmonization remains a highly complex process due to variation of national regulations that are based on national customs, culture, ethics, religion and other beliefs [6]. Harmonization of laws is designed to incorporate different legal systems under a basic framework. To overcome the highly complex process of harmonization in the area of research, it has been suggested to combine similarities between legislations and regulations of countries under a basic framework like a European research directive. A framework should acknowledge these local cultural or religious beliefs, as disregarding them is neither feasible nor desirable. While the desirable goal of harmonizing regulation will certainly benefit research in the future, both IRBs and researchers will have to put in efforts until that time. IRBs can accelerate the turnover by only requiring central IRB approval and researchers should respond quicker and more comprehensively to questions from IRBs, preventing the repetition of questions.

\section{Strengths and limitations}

The CENTER-TBI study provides a unique opportunity to provide comprehensive insight in the procedural differences between European IRBs. The study benefits from its large size and because the data acquisition process increased the quality and completeness of documents. Despite the quality of the documents, results were still dependent on the recorded information. Therefore, we could not always identify causal factors for variation, which is something to look for in future initiatives. The data on IRB review procedures in an observational study conducted with mentally incapacitated patients in neurotrauma centers might not be generalizable for other research settings.

\section{Conclusions}

This study shows variation between IRB procedures across Europe, which pose major challenges to large European research collaborations. Differences are likely caused by the lack of harmonization, integration and implementation of national legislations and regulations. To optimize efficiency for multinational European studies in context of obtaining IRB approval, the encountered differences and inefficiencies should be studied further and policymakers should evaluate the opportunities to optimize regulatory harmonization, while acknowledging the boundaries of national sovereignty and local cultural preferences.

\section{Supplementary information}

Supplementary information accompanies this paper at https://doi.org/10. 1186/s12910-020-00480-8.

\section{Supplementary files.}

\section{Abbreviations}

EU: European Union; CENTER-TBI: Collaborative European NeuroTrauma Effectiveness Research in Traumatic Brain Injury; CER: Comparative Effectiveness Research; GDPR: General Data Protection Regulation; ICON: ICON plc; IRB: Institutional Review Board; M: Missing; PHD: Personal Health related Data; TBI: Traumatic Brain Injury; UK: United Kingdom

\section{Acknowledgements}

Collaborating authors:

The CENTER-TBI participants and investigators:

Cecilia Åkerlund ${ }^{1}$, Krisztina Amrein ${ }^{2}$, Nada Andelic ${ }^{3}$, Lasse Andreassen ${ }^{4}$, Audny Anke ${ }^{5}$, Anna Antoni ${ }^{6}$, Gérard Audibert ${ }^{7}$, Philippe Azouvi ${ }^{8}$, Maria Luisa Azzolini ${ }^{9}$, Ronald Bartels ${ }^{10}$, Pál Barzó ${ }^{11}$, Romuald Beauvais ${ }^{12}$, Ronny Beer ${ }^{13}$, BoMichael Bellander ${ }^{14}$, Antonio Belli ${ }^{15}$, Habib Benali ${ }^{16}$, Maurizio Berardino ${ }^{17}$, Luigi Beretta $^{9}$, Morten Blaabjerg ${ }^{18}$, Peter Bragge ${ }^{19}$, Alexandra Brazinova ${ }^{20}$, Vibeke Brinck ${ }^{21}$, Joanne Brooker ${ }^{22}$, Camilla Brorsson ${ }^{23}$, Andras Buki $^{24}$, Monika Bullinger $^{25}$, Manuel Cabeleira ${ }^{26}$, Alessio Caccioppola ${ }^{27}$, Emiliana Calappi ${ }^{27}$, Maria Rosa Calvi ${ }^{9}$, Peter Cameron ${ }^{28}$, Guillermo Carbayo Lozano ${ }^{29}$, Marco Carbonara ${ }^{27}$, Simona Cavallo ${ }^{17}$, Giorgio Chevallard ${ }^{30}$, Arturo Chieregato ${ }^{30}$, Giuseppe Citerio ${ }^{31,32}$, Iris Ceyisakar ${ }^{33}$, Mark Coburn ${ }^{34}$, Jonathan Coles ${ }^{35}$, Jamie D. Cooper $^{36}$, Marta Correia ${ }^{37}$, Amra Čović ${ }^{38}$, Nicola Curry ${ }^{39}$, Endre Czeiter ${ }^{24}$, Marek Czosnyka ${ }^{26}$, Claire Dahyot-Fizelier ${ }^{40}$, Paul Dark ${ }^{41}$, Helen Dawes ${ }^{42}$, 'Véronique De Keyser ${ }^{43}$, Vincent Degos ${ }^{16}$, Francesco Della Corte ${ }^{44}$, Hugo den Boogert $^{10}$, Bart Depreitere ${ }^{45}$, Đula Đilvesi ${ }^{46}$, Abhishek Dixit ${ }^{47}$, Emma Donoghue $^{22}$ Jens Dreier ${ }^{48}$, Guy-Loup Dulière ${ }^{49}$, Ari Ercole ${ }^{47}$, Patrick Esser ${ }^{42}$, Erzsébet Ezer ${ }^{50}$, Martin Fabricius ${ }^{51}$, Valery L. Feigin ${ }^{52}$, Kelly Foks ${ }^{53}$, Shirin Frisvold ${ }^{54}$, Alex Furmanov $^{55}$, Pablo Gagliardo ${ }^{56}$, Damien Galanaud ${ }^{16}$, Dashiell Gantner ${ }^{28}$, Guoyi $\mathrm{Gao}^{57}$, Pradeep George ${ }^{58}$, Alexandre Ghuysen ${ }^{59}$, Lelde Giga ${ }^{60}$, Ben Glocker ${ }^{61}$, Jagoš Golubovic ${ }^{46}$, Pedro A. Gomez ${ }^{62}$, Johannes Gratz ${ }^{63}$, Benjamin Gravesteijn ${ }^{33}$, Francesca Grossi ${ }^{44}$, Russell L. Gruen ${ }^{64}$, Deepak Gupta ${ }^{65}$, Juanita A. Haagsma $^{33}$, lain Haitsma ${ }^{66}$, Raimund Helbok ${ }^{13}$, Eirik Helseth ${ }^{67}$, Lindsay Horton 68 , Jilske Huijben ${ }^{33}$, Peter J. Hutchinson ${ }^{69}$, Bram Jacobs ${ }^{70}$, Stefan Jankowski ${ }^{71}$, Mike Jarrett ${ }^{21}$, Ji-yao Jiang ${ }^{57}$, Faye Johnson ${ }^{72}$, Kelly Jones ${ }^{52}$, Mladen Karan ${ }^{46}$, Angelos G. Kolias ${ }^{69}$, Erwin Kompanje ${ }^{73}$, Daniel Kondziella ${ }^{51}$, Evgenios Koraropoulos $^{47}$, Lars-Owe Koskinen ${ }^{74}$, Noémi Kovács ${ }^{75}$, Ana Kowark ${ }^{34}$, Alfonso Lagares $^{62}$, Linda Lanyon ${ }^{58}$, Steven Laureys ${ }^{76}$, Fiona Lecky ${ }^{77,}$, ${ }^{78}$, Didier Ledoux ${ }^{76}$, Rolf Lefering ${ }^{79}$, Valerie Legrand ${ }^{80}$, Aurelie Lejeune ${ }^{81}$, Leon Levi ${ }^{82}$, Roger Lightfoot $^{83}$, Hester Lingsma ${ }^{33}$, Andrew I.R. Maas ${ }^{43}$, Ana M. Castaño-León ${ }^{62}$, Marc Maegele ${ }^{84}$, Marek Majdan ${ }^{20}$, Alex Manara ${ }^{85}$, Geoffrey Manley ${ }^{86}$, Costanza Martino ${ }^{87}$, Hugues Maréchal ${ }^{49}$, Julia Mattern ${ }^{88}$, Catherine McMahonn ${ }^{89}$, Béla Melegh ${ }^{90}$, David Menon ${ }^{47}$, Tomas Menovsky ${ }^{43}$, Benoit Misset ${ }^{76}$, Davide $^{\prime}$ Mulazzi $^{27}$, Visakh Muraleedharan ${ }^{58}$, Lynnette Murray ${ }^{28}$, Ancuta Negru ${ }^{91}$, David Nelson ${ }^{1}$, Virginia Newcombe ${ }^{47}$, Daan Nieboer ${ }^{33}$, József Nyirádi ${ }^{2}$, Otesile Olubukola $^{77}$, Matej Oresic ${ }^{92}$, Fabrizio Ortolano ${ }^{27}$, Aarno Palotie ${ }^{93,94,95}$, Paul M. Parizel $^{96}$, Jean-François Payen ${ }^{97}$, Natascha Perera ${ }^{12}$, Vincent Perlbarg ${ }^{16}$, Paolo Persona $^{98}$, Wilco Peul ${ }^{99}$, Anna Piippo-Karjalainen ${ }^{100}$, Matti Pirinen ${ }^{93}$, Horia Ples $^{91}$, Suzanne Polinder ${ }^{33}$, Inigo Pomposo ${ }^{29}$, Jussi P. Posti ${ }^{101}$, Louis Puybasset $^{102}$, Andreea Radoi ${ }^{103}$, Arminas Ragauskas ${ }^{104}$, Rahul Raj ${ }^{100}$, Malinka Rambadagalla $^{105}$, Jonathan Rhodes ${ }^{106}$, Sylvia Richardson ${ }^{107}$, Sophie Richter ${ }^{47}$, Samuli Ripatti ${ }^{93}$, Saulius Rocka ${ }^{104}$, Cecilie Roe ${ }^{108}$, Olav Roise ${ }^{109,110}$, Jonathan Rosand ${ }^{111}$, Jeffrey V. Rosenfeld ${ }^{112}$, Christina Rosenlund ${ }^{113}$, Guy Rosenthal ${ }^{55}$, Rolf Rossaint ${ }^{34}$, Sandra Rossi ${ }^{98}$, Daniel Rueckert ${ }^{61}$, Martin Rusnák ${ }^{114}$, Juan Sahuquillo ${ }^{103}$, Oliver Sakowitz ${ }^{88,115}$, Renan Sanchez-Porras ${ }^{115}$, Janos Sandor $^{116}$, Nadine Schäfer ${ }^{79}$, Silke Schmidt ${ }^{117}$, Herbert Schoechl ${ }^{118}$, Guus Schoonman $^{119}$, Rico Frederik Schou ${ }^{120}$, Elisabeth Schwendenwein ${ }^{6}$, Charlie Sewalt ${ }^{33}$, Toril Skandsen ${ }^{121,122}$, Peter Smielewski ${ }^{26}$, Abayomi Sorinola ${ }^{123}$, Emmanuel Stamatakis $^{47}$, Simon Stanworth ${ }^{39}$, Robert Stevens ${ }^{124}$, William Stewart ${ }^{125}$, Ewout W. Steyerberg ${ }^{33,126}$, Nino Stocchetti ${ }^{127}$, Nina Sundström ${ }^{128}$, Anneliese Synnot $^{22,}{ }^{129}$, Riikka Takala ${ }^{130}$, Viktória Tamás ${ }^{123}$, Tomas Tamosuitis ${ }^{131}$, Mark Steven Taylor ${ }^{20}$, Braden Te Ao ${ }^{52}$, Olli Tenovuo ${ }^{101}$, Alice Theadom ${ }^{52}$, Matt Thomas $^{85}$, Dick Tibboel ${ }^{132}$, Marjolein Timmers ${ }^{73}$, Christos Tolias ${ }^{133}$, Tony Trapani $^{28}$, Cristina Maria Tudora ${ }^{91}$, Peter Vajkoczy ${ }^{134}$, Shirley Vallance ${ }^{28}$, Egils Valeinis $^{60}$, Zoltán Vámos ${ }^{50}$, Gregory Van der Steen ${ }^{43}$, Joukje van der Naalt ${ }^{70}$, Jeroen T.J.M. van Dijck ${ }^{99}$, Thomas A. van Essen ${ }^{99}$, Wim Van Hecke ${ }^{135}$, Caroline 
van Heugten ${ }^{136}$, Dominique Van Praag ${ }^{137}$, Thijs Vande Vyvere ${ }^{135}$, Roel P. J. van Wijk $^{99}$, Alessia Vargiolu' ${ }^{32}$, Emmanuel Vega ${ }^{81}$, Kimberley Velt ${ }^{33}$, Jan Verheyden ${ }^{135}$, Paul M. Vespa ${ }^{138}$, Anne Vik ${ }^{120,}{ }^{139}$, Rimantas Vilcinis ${ }^{131}$, Victor Volo$v_{\text {vici }}{ }^{66}$, Nicole von Steinbüchel ${ }^{38}$, Daphne Voormolen ${ }^{33}$, Petar Vulekovic ${ }^{46}$, Kevin K.W. Wang ${ }^{140}$, Eveline Wiegers ${ }^{33}$, Guy Williams ${ }^{47}$, Lindsay Wilson ${ }^{68}$, Stefan Winzeck ${ }^{47}$, Stefan Wolf ${ }^{141}$, Zhihui Yang ${ }^{140}$, Peter Ylén ${ }^{142}$, Alexander Younsi ${ }^{88}$, Frederick A. Zeiler ${ }^{47,143}$, Veronika Zelinkova ${ }^{20}$, Agate Ziverte ${ }^{60}$, Tommaso Zoerle ${ }^{27}$.

${ }^{1}$ Department of Physiology and Pharmacology, Section of Perioperative Medicine and Intensive Care, Karolinska Institutet, Stockholm, Sweden.

2 János Szentágothai Research Centre, University of Pécs, Pécs, Hungary.

${ }^{3}$ Division of Surgery and Clinical Neuroscience, Department of Physical Medicine and Rehabilitation, Oslo University Hospital and University of Oslo, Oslo, Norway.

${ }^{4}$ Department of Neurosurgery, University Hospital Northern Norway, Tromso, Norway.

${ }^{5}$ Department of Physical Medicine and Rehabilitation, University Hospital Northern Norway, Tromso, Norway.

${ }^{6}$ Trauma Surgery, Medical University Vienna, Vienna, Austria.

${ }^{7}$ Department of Anesthesiology \& Intensive Care, University Hospital Nancy, Nancy, France.

${ }^{8}$ Raymond Poincare hospital, Assistance Publique - Hopitaux de Paris, Paris, France.

${ }^{9}$ Department of Anesthesiology \& Intensive Care, S Raffaele University Hospital, Milan, Italy.

${ }^{10}$ Department of Neurosurgery, Radboud University Medical Center,

Nijmegen, The Netherlands.

${ }_{11}$ Department of Neurosurgery, University of Szeged, Szeged, Hungary.

12 International Projects Management, ARTTIC, Munchen, Germany.

${ }^{13}$ Department of Neurology, Neurological Intensive Care Unit, Medical University of Innsbruck, Innsbruck, Austria.

${ }^{14}$ Department of Neurosurgery \& Anesthesia \& intensive care medicine, Karolinska University Hospital, Stockholm, Sweden.

${ }^{15} \mathrm{NIHR}$ Surgical Reconstruction and Microbiology Research Centre, Birmingham, UK.

${ }^{16}$ Anesthesie-Réanimation, Assistance Publique - Hopitaux de Paris, Paris, France.

${ }^{17}$ Department of Anesthesia \& ICU, AOU Città della Salute e della Scienza di Torino - Orthopedic and Trauma Center, Torino, Italy.

${ }^{18}$ Department of Neurology, Odense University Hospital, Odense, Denmark.

${ }^{19}$ BehaviourWorks Australia, Monash Sustainability Institute, Monash University, Victoria, Australia.

20 Department of Public Health, Faculty of Health Sciences and Social Work, Trnava University, Trnava, Slovakia.

${ }_{21}^{21}$ Quesgen Systems Inc., Burlingame, California, USA.

22 Australian \& New Zealand Intensive Care Research Centre, Department of Epidemiology and Preventive Medicine, School of Public Health and Preventive Medicine, Monash University, Melbourne, Australia.

${ }^{23}$ Department of Surgery and Perioperative Science, Umeå University, Umeå, Sweden.

${ }^{24}$ Department of Neurosurgery, Medical School, University of Pécs, Hungary and Neurotrauma Research Group, János Szentágothai Research Centre, University of Pécs, Hungary.

${ }^{25}$ Department of Medical Psychology, Universitätsklinikum HamburgEppendorf, Hamburg, Germany.

${ }^{26}$ Brain Physics Lab, Division of Neurosurgery, Dept of Clinical Neurosciences, University of Cambridge, Addenbrooke's Hospital, Cambridge, UK.

${ }^{27}$ Neuro ICU, Fondazione IRCCS Cà Granda Ospedale Maggiore Policlinico, Milan, Italy.

${ }^{28}$ ANZIC Research Centre, Monash University, Department of Epidemiology and Preventive Medicine, Melbourne, Victoria, Australia.

${ }^{29}$ Department of Neurosurgery, Hospital of Cruces, Bilbao, Spain.

${ }^{30}$ Neurolntensive Care, Niguarda Hospital, Milan, Italy.

${ }^{31}$ School of Medicine and Surgery, Università Milano Bicocca, Milano, Italy.

32 Neurolntensive Care, ASST di Monza, Monza, Italy.

33 Department of Public Health, Erasmus Medical Center-University Medical Center, Rotterdam, The Netherlands.

${ }^{34}$ Department of Anaesthesiology, University Hospital of Aachen, Aachen, Germany.

35 Department of Anesthesia \& Neurointensive Care, Cambridge University Hospital NHS Foundation Trust, Cambridge, UK.
${ }^{36}$ School of Public Health \& PM, Monash University and The Alfred Hospital, Melbourne, Victoria, Australia.

${ }^{37}$ Radiology/MRI department, MRC Cognition and Brain Sciences Unit, Cambridge, UK.

38 Institute of Medical Psychology and Medical Sociology, Universitätsmedizin Göttingen, Göttingen, Germany.

${ }^{39}$ Oxford University Hospitals NHS Trust, Oxford, UK.

${ }^{40}$ Intensive Care Unit, CHU Poitiers, Potiers, France.

${ }^{41}$ University of Manchester NIHR Biomedical Research Centre, Critical Care Directorate, Salford Royal Hospital NHS Foundation Trust, Salford, UK.

${ }^{42}$ Movement Science Group, Faculty of Health and Life Sciences, Oxford

Brookes University, Oxford, UK.

${ }^{43}$ Department of Neurosurgery, Antwerp University Hospital and University of Antwerp, Edegem, Belgium.

${ }^{44}$ Department of Anesthesia \& Intensive Care, Maggiore Della Carità Hospital, Novara, Italy.

${ }^{45}$ Department of Neurosurgery, University Hospitals Leuven, Leuven, Belgium.

${ }^{46}$ Department of Neurosurgery, Clinical centre of Vojvodina, Faculty of

Medicine, University of Novi Sad, Novi Sad, Serbia.

${ }^{47}$ Division of Anaesthesia, University of Cambridge, Addenbrooke's Hospital, Cambridge, UK.

${ }^{48}$ Center for Stroke Research Berlin, Charité - Universitätsmedizin Berlin, corporate member of Freie Universität Berlin, Humboldt-Universität zu Berlin, and Berlin Institute of Health, Berlin, Germany.

${ }^{49}$ Intensive Care Unit, CHR Citadelle, Liège, Belgium.

${ }^{50}$ Department of Anaesthesiology and Intensive Therapy, University of Pécs, Pécs, Hungary.

${ }^{51}$ Departments of Neurology, Clinical Neurophysiology and Neuroanesthesiology, Region Hovedstaden Rigshospitalet, Copenhagen, Denmark.

${ }^{52}$ National Institute for Stroke and Applied Neurosciences, Faculty of Health and Environmental Studies, Auckland University of Technology, Auckland,

New Zealand.

${ }^{53}$ Department of Neurology, Erasmus MC, Rotterdam, the Netherlands.

${ }^{54}$ Department of Anesthesiology and Intensive care, University Hospital Northern Norway, Tromso, Norway.

${ }^{55}$ Department of Neurosurgery, Hadassah-hebrew University Medical center, Jerusalem, Israel.

${ }^{56}$ Fundación Instituto Valenciano de Neurorrehabilitación (FIVAN), Valencia, Spain.

${ }^{57}$ Department of Neurosurgery, Shanghai Renji hospital, Shanghai Jiaotong

University/school of medicine, Shanghai, China.

${ }^{58}$ Karolinska Institutet, INCF International Neuroinformatics Coordinating

Facility, Stockholm, Sweden.

${ }^{59}$ Emergency Department, $\mathrm{CHU}$, Liège, Belgium.

${ }^{60}$ Neurosurgery clinic, Pauls Stradins Clinical University Hospital, Riga, Latvia.

${ }^{61}$ Department of Computing, Imperial College London, London, UK.

${ }^{62}$ Department of Neurosurgery, Hospital Universitario 12 de Octubre, Madrid, Spain.

${ }^{63}$ Department of Anesthesia, Critical Care and Pain Medicine, Medical University of Vienna, Austria.

${ }^{64}$ College of Health and Medicine, Australian National University, Canberra, Australia.

${ }^{65}$ Department of Neurosurgery, Neurosciences Centre \& JPN Apex trauma centre, All India Institute of Medical Sciences, New Delhi-110029, India.

${ }^{66}$ Department of Neurosurgery, Erasmus MC, Rotterdam, the Netherlands.

${ }^{67}$ Department of Neurosurgery, Oslo University Hospital, Oslo, Norway.

${ }^{68}$ Division of Psychology, University of Stirling, Stirling, UK.

${ }^{69}$ Division of Neurosurgery, Department of Clinical Neurosciences, Addenbrooke's Hospital \& University of Cambridge, Cambridge, UK.

${ }^{70}$ Department of Neurology, University of Groningen, University Medical Center Groningen, Groningen, Netherlands.

${ }^{71}$ Neurointensive Care, Sheffield Teaching Hospitals NHS Foundation Trust, Sheffield, UK.

${ }^{72}$ Salford Royal Hospital NHS Foundation Trust Acute Research Delivery Team, Salford, UK.

${ }^{73}$ Department of Intensive Care and Department of Ethics and Philosophy of Medicine, Erasmus Medical Center, Rotterdam, The Netherlands.

${ }^{74}$ Department of Clinical Neuroscience, Neurosurgery, Umeå University, Umeå, Sweden. 
${ }^{75}$ Hungarian Brain Research Program - Grant No. KTIA_13_NAP-A-II/8, University of Pécs, Pécs, Hungary.

${ }^{76}$ Cyclotron Research Center, University of Liège, Liège, Belgium.

${ }^{77}$ Centre for Urgent and Emergency Care Research (CURE), Health Services Research Section, School of Health and Related Research (ScHARR), University of Sheffield, Sheffield, UK.

${ }^{78}$ Emergency Department, Salford Royal Hospital, Salford UK.

79 Institute of Research in Operative Medicine (IFOM), Witten/Herdecke University, Cologne, Germany.

${ }^{80}$ VP Global Project Management CNS, ICON, Paris, France.

${ }^{81}$ Department of Anesthesiology-Intensive Care, Lille University Hospital, Lille, France.

82 Department of Neurosurgery, Rambam Medical Center, Haifa, Israel.

${ }^{83}$ Department of Anesthesiology \& Intensive Care, University Hospitals Southhampton NHS Trust, Southhampton, UK.

${ }^{84}$ Cologne-Merheim Medical Center (CMMC), Department of Traumatology, Orthopedic Surgery and Sportmedicine, Witten/Herdecke University, Cologne, Germany.

${ }^{85}$ Intensive Care Unit, Southmead Hospital, Bristol, Bristol, UK.

${ }^{86}$ Department of Neurological Surgery, University of California, San Francisco,

California, USA.

${ }^{87}$ Department of Anesthesia \& Intensive Care,M. Bufalini Hospital, Cesena, Italy.

88 Department of Neurosurgery, University Hospital Heidelberg, Heidelberg, Germany.

${ }^{89}$ Department of Neurosurgery, The Walton centre NHS Foundation Trust, Liverpool, UK

${ }^{90}$ Department of Medical Genetics, University of Pécs, Pécs, Hungary.

${ }^{91}$ Department of Neurosurgery, Emergency County Hospital Timisoara, Timisoara, Romania.

${ }^{92}$ School of Medical Sciences, Örebro University, Örebro, Sweden.

93 Institute for Molecular Medicine Finland, University of Helsinki, Helsinki, Finland.

${ }^{94}$ Analytic and Translational Genetics Unit, Department of Medicine; Psychiatric \& Neurodevelopmental Genetics Unit, Department of Psychiatry; Department of Neurology, Massachusetts General Hospital, Boston, MA, USA.

${ }^{95}$ Program in Medical and Population Genetics; The Stanley Center for Psychiatric Research, The Broad Institute of MIT and Harvard, Cambridge, MA, USA.

${ }^{96}$ Department of Radiology, University of Antwerp, Edegem, Belgium

${ }^{97}$ Department of Anesthesiology \& Intensive Care, University Hospital of Grenoble, Grenoble, France.

98 Department of Anesthesia \& Intensive Care, Azienda Ospedaliera Università di Padova, Padova, Italy.

99 Dept. of Neurosurgery, Leiden University Medical Center, Leiden, The Netherlands and Dept. of Neurosurgery, Medical Center Haaglanden, The Hague, The Netherlands.

100 Department of Neurosurgery, Helsinki University Central Hospital.

101 Division of Clinical Neurosciences, Department of Neurosurgery and Turku Brain Injury Centre, Turku University Hospital and University of Turku, Turku, Finland.

102 Department of Anesthesiology and Critical Care, Pitié -Salpêtrière Teaching Hospital, Assistance Publique, Hôpitaux de Paris and University Pierre et Marie Curie, Paris, France.

${ }^{103}$ Neurotraumatology and Neurosurgery Research Unit (UNINN), Vall d'Hebron Research Institute, Barcelona, Spain.

${ }^{104}$ Department of Neurosurgery, Kaunas University of technology and Vilnius University, Vilnius, Lithuania.

105 Department of Neurosurgery, Rezekne Hospital, Latvia.

106 Department of Anaesthesia, Critical Care \& Pain Medicine NHS Lothian \& University of Edinburg, Edinburgh, UK.

${ }^{107}$ Director, MRC Biostatistics Unit, Cambridge Institute of Public Health, Cambridge, UK.

${ }^{108}$ Department of Physical Medicine and Rehabilitation, Oslo University Hospital/University of Oslo, Oslo, Norway.

${ }^{109}$ Division of Orthopedics, Oslo University Hospital, Oslo, Norway.

110 Institue of Clinical Medicine, Faculty of Medicine, University of Oslo, Oslo, Norway.

${ }^{111}$ Broad Institute, Cambridge MA Harvard Medical School, Boston MA, Massachusetts General Hospital, Boston MA, USA.
${ }^{112}$ National Trauma Research Institute, The Alfred Hospital, Monash University, Melbourne, Victoria, Australia.

${ }^{113}$ Department of Neurosurgery, Odense University Hospital, Odense, Denmark.

${ }^{114}$ International Neurotrauma Research Organisation, Vienna, Austria.

115 Klinik für Neurochirurgie, Klinikum Ludwigsburg, Ludwigsburg, Germany.

116 Division of Biostatistics and Epidemiology, Department of Preventive

Medicine, University of Debrecen, Debrecen, Hungary.

117 Department Health and Prevention, University Greifswald, Greifswald, Germany.

${ }^{118}$ Department of Anaesthesiology and Intensive Care, AUVA Trauma Hospital, Salzburg, Austria.

119 Department of Neurology, Elisabeth-TweeSteden Ziekenhuis, Tilburg, the Netherlands.

${ }^{120}$ Department of Neuroanesthesia and Neurointensive Care, Odense University Hospital, Odense, Denmark.

121 Department of Neuromedicine and Movement Science, Norwegian University of Science and Technology, NTNU, Trondheim, Norway.

122 Department of Physical Medicine and Rehabilitation, St. Olavs Hospital,

Trondheim University Hospital, Trondheim, Norway.

${ }^{123}$ Department of Neurosurgery, University of Pécs, Pécs, Hungary.

${ }^{124}$ Division of Neuroscience Critical Care, John Hopkins University School of Medicine, Baltimore, USA.

125 Department of Neuropathology, Queen Elizabeth University Hospital and University of Glasgow, Glasgow, UK.

${ }^{126}$ Dept. of Department of Biomedical Data Sciences, Leiden University Medical Center, Leiden, The Netherlands.

127 Department of Pathophysiology and Transplantation, Milan University, and Neuroscience ICU, Fondazione IRCCS Cà Granda Ospedale Maggiore Policlinico, Milano, Italy.

${ }^{128}$ Department of Radiation Sciences, Biomedical Engineering, Umeå University, Umeå, Sweden.

${ }^{129}$ Cochrane Consumers and Communication Review Group, Centre for Health Communication and Participation, School of Psychology and Public Health, La Trobe University, Melbourne, Australia.

130 Perioperative Services, Intensive Care Medicine and Pain Management, Turku University Hospital and University of Turku, Turku, Finland.

${ }^{131}$ Department of Neurosurgery, Kaunas University of Health Sciences, Kaunas, Lithuania.

132 Intensive Care and Department of Pediatric Surgery, Erasmus Medical Center, Sophia Children's Hospital, Rotterdam, The Netherlands.

133 Department of Neurosurgery, Kings college London, London, UK.

${ }^{134}$ Neurologie, Neurochirurgie und Psychiatrie, Charité - Universitätsmedizin Berlin, Berlin, Germany.

135 icoMetrix NV, Leuven, Belgium.

${ }^{136}$ Movement Science Group, Faculty of Health and Life Sciences, Oxford Brookes University, Oxford, UK.

137 Psychology Department, Antwerp University Hospital, Edegem, Belgium.

${ }^{138}$ Director of Neurocritical Care, University of California, Los Angeles, USA.

139 Department of Neurosurgery, St. Olavs Hospital, Trondheim University Hospital, Trondheim, Norway.

140 Department of Emergency Medicine, University of Florida, Gainesville, Florida, USA.

${ }^{141}$ Department of Neurosurgery, Charité - Universitätsmedizin Berlin, corporate member of Freie Universität Berlin, Humboldt-Universität zu Berlin, and Berlin Institute of Health, Berlin, Germany.

142 VIT Technical Research Centre, Tampere, Finland.

${ }^{143}$ Section of Neurosurgery, Department of Surgery, Rady Faculty of Health Sciences, University of Manitoba, Winnipeg, MB, Canada.

\begin{tabular}{lll}
\hline Åkerlund & Cecilia & cecilia.ai.akerlund@gmail.com \\
\hline Amrein & Krisztina & tina.amrein84@gmail.com \\
Andelic & Nada & NADAND@ous-hf.no \\
Andreassen & Lasse & Lasse.Andreassen@unn.no \\
Anke & Audny & Audny.anke@unn.no \\
Antoni & Anna & anna.antoni@meduniwien.ac.at
\end{tabular}


(Continued)

\begin{tabular}{|c|c|c|}
\hline Åkerlund & Cecilia & cecilia.ai.akerlund@gmail.com \\
\hline Audibert & Gérard & g.audibert@chu-nancy.fr \\
\hline Azouvi & Philippe & philippe.azouvi@rpc.aphp.fr \\
\hline Azzolini & Maria Luisa & azzolini.marialuisa@hsr.it \\
\hline Bartels & Ronald & Ronald.Bartels@radboudumc.nl \\
\hline Barzó & Pál & pbarzo@gmail.com \\
\hline Beauvais & Romuald & beauvais@arttic.eu \\
\hline Beer & Ronny & ronny.beer@i-med.ac.at \\
\hline Bellander & Bo-Michael & bo-michael.bellander@karolinska.se \\
\hline Belli & Antonio & a.belli@bham.ac.uk \\
\hline Benali & Habib & habib.benali@gmail.com \\
\hline Berardino & Maurizio & maurizio_berardino@fastwebnet.it \\
\hline Beretta & Luigi & beretta.luigi@hsr.it \\
\hline Blaabjerg & Morten & morten.blaabjerg1@rsyd.dk \\
\hline Bragge & Peter & peter.bragge@monash.edu \\
\hline Brazinova & Alexandra & alexandra.brazinova@gmail.com \\
\hline Brinck & Vibeke & vibeke.brinck@quesgen.com \\
\hline Brooker & Joanne & Joanne.Brooker@monash.edu \\
\hline Brorsson & Camilla & Camilla.Brorsson@umu.se \\
\hline Buki & Andras & 2saturn@gmail.com \\
\hline Bullinger & Monika & bullinger@uke.de \\
\hline Cabeleira & Manuel & mc916@cam.ac.uk \\
\hline Caccioppola & Alessio & alessio.caccioppola@gmail.com \\
\hline Calappi & Emiliana & calemy02@yahoo.it \\
\hline Calvi & Maria Rosa & calvi.mariarosa@hsr.it \\
\hline Cameron & Peter & peter.cameron@med.monash.edu.au \\
\hline Carbayo Lozano & Guillermo & guillermobilbo@gmail.com \\
\hline Carbonara & Marco & marco.carbonara@gmail.com \\
\hline Castaño-León & Ana $M$. & ana.maria.castano.leon@gmail.com \\
\hline Cavallo & Simona & cavallosimona1@gmail.com \\
\hline Chevallard & Giorgio & giorgio.chevallard@ospedaleniguarda.it \\
\hline Chieregato & Arturo & arturo.chieregato@ospedaleniguarda.it \\
\hline Citerio & Giuseppe & giuseppe.citerio@unimib.it \\
\hline Ceyisakar & Iris & i.ceyisakar@erasmusmc.nl \\
\hline Coburn & Mark Steven & mcoburn@ukaachen.de \\
\hline Coles & Jonathan & jpc44@wbic.cam.ac.uk \\
\hline Cooper & Jamie D. & jamie.cooper@monash.edu \\
\hline Correia & Marta & Marta.Correia@mrc-cbu.cam.ac.uk \\
\hline Čović & Amra & amra.covic@med.uni-goettingen.de \\
\hline Curry & Nicola & nicola.curry@ouh.nhs.uk \\
\hline Czeiter & Endre & endre.czeiter@gmail.com \\
\hline Czosnyka & Marek & mc141@medschl.cam.ac.uk \\
\hline Dahyot-Fizelier & Claire & c.dahyot-fizelier@chu-poitiers.fr \\
\hline Dark & Paul & paul.m.dark@manchester.ac.uk \\
\hline Dawes & Helen & hdawes@brookes.ac.uk \\
\hline
\end{tabular}

(Continued)

\begin{tabular}{|c|c|c|}
\hline Åkerlund & Cecilia & cecilia.ai.akerlund@gmail.com \\
\hline De Keyser & Véronique & veronique.dekeyser@uza.be \\
\hline Degos & Vincent & vincent.degos@aphp.fr \\
\hline Della Corte & Francesco & dellacorte.f@gmail.com \\
\hline den Boogert & Hugo & Hugo.denBoogert@radboudumc.nl \\
\hline Depreitere & Bart & bart.depreitere@uzleuven.be \\
\hline Đilvesi & Đula & djuladjilvesi@gmail.com \\
\hline Dixit & Abhishek & ad825@cam.ac.uk \\
\hline Donoghue & Emma & emma.donoghue@monash.edu \\
\hline Dreier & Jens & jens.dreier@charite.de \\
\hline Dulière & Guy-Loup & glduliere@gmail.com \\
\hline Ercole & Ari & ae105@cam.ac.uk \\
\hline Esser & Patrick & pesser@brookes.ac.uk \\
\hline Ezer & Erzsébet & ezererzsebet@yahoo.com \\
\hline Fabricius & Martin & fabricius@dadlnet.dk \\
\hline Feigin & Valery L. & valery.feigin@aut.ac.nz \\
\hline Foks & Kelly & k.foks@erasmusmc.nl \\
\hline Frisvold & Shirin & Shirin.Kordasti@unn.no \\
\hline Furmanov & Alex & alexpuil@yahoo.com \\
\hline Gagliardo & Pablo & pablog@fivan.org \\
\hline Galanaud & Damien & galanaud@gmail.com \\
\hline Gantner & Dashiell & dashiell.gantner@monash.edu \\
\hline Gao & Guoyi & gao3@sina.com \\
\hline George & Pradeep & george@incf.org \\
\hline Ghuysen & Alexandre & A.Ghuysen@chu.ulg.ac.be \\
\hline Giga & Lelde & lelde.giga@stradini.lv \\
\hline Glocker & Ben & b.glocker@imperial.ac.uk \\
\hline Golubović & Jagoš & jagosgolubovic@gmail.com \\
\hline Gomez & Pedro A. & pagolopez@gmail.com \\
\hline Gratz & Johannes & johannes.gratz@meduniwien.ac.at \\
\hline Gravesteijn & Benjamin & b.gravesteijn@erasmusmc.nl \\
\hline Grossi & Francesca & francesca.grossi@libero.it \\
\hline Gruen & Russell L. & russell.gruen@anu.edu.au \\
\hline Gupta & Deepak & drdeepakgupta@gmail.com \\
\hline Haagsma & Juanita A. & j.haagsma@erasmusmc.nl \\
\hline Haitsma & lain & i.haitsma@erasmusmc.nl \\
\hline Helbok & Raimund & Raimund.Helbok@tirol-kliniken.at \\
\hline Helseth & Eirik & EHELSETH@ous-hf.no \\
\hline Horton & Lindsay & lindsay.horton@stir.ac.uk \\
\hline Huijben & Jilske & j.a.huijben@erasmusmc.nl \\
\hline Hutchinson & Peter J. & pjah2@cam.ac.uk \\
\hline Jacobs & Bram & b.jacobs@umcg.nl \\
\hline Jankowski & Stefan & Stefan.Jankowski@sth.nhs.uk \\
\hline Jarrett & Mike & mike.jarrett@quesgen.com \\
\hline Jiang & Ji-yao & jiyaojiang@126.com \\
\hline
\end{tabular}


(Continued)

\begin{tabular}{|c|c|c|}
\hline Åkerlund & Cecilia & cecilia.ai.akerlund@gmail.com \\
\hline Johnson & Faye & faye.johnson@live.co.uk \\
\hline Jones & Kelly & kejones@aut.ac.nz \\
\hline Karan & Mladen & mladjokaran@gmail.com \\
\hline Kolias & Angelos G. & angeloskolias@gmail.com \\
\hline Kompanje & Erwin & erwinkompanje@me.com \\
\hline Kondziella & Daniel & Daniel.Kondziella@regionh.dk \\
\hline Koraropoulos & Evgenios & ek481@cam.ac.uk \\
\hline Koskinen & Lars-Owe & Lars-Owe.Koskinen@umu.se \\
\hline Kovács & Noémi & kovacs.noemi@pte.hu \\
\hline Lagares & Alfonso & algadoc@yahoo.com \\
\hline Lanyon & Linda & lindal@incf.org \\
\hline Laureys & Steven & steven.laureys@ulg.ac.be \\
\hline Lecky & Fiona & f.e.lecky@sheffield.ac.uk \\
\hline Ledoux & Didier & dledoux@chu.ulg.ac.be \\
\hline Lefering & Rolf & Rolf.Lefering@uni-wh.de \\
\hline Legrand & Valerie & Valerie.Legrand@iconplc.com \\
\hline Lejeune & Aurelie & aurelie.lejeune@chru-lille.fr \\
\hline Levi & Leon & Ilevi@rambam.health.gov.ll \\
\hline Lightfoot & Roger & Roger.Lightfoot@uhs.nhs.uk \\
\hline Lingsma & Hester & h.lingsma@erasmusmc.nl \\
\hline Maas & Andrew I.R. & andrew.maas@uza.be \\
\hline Maegele & Marc & Marc.Maegele@t-online.de \\
\hline Majdan & Marek & mmajdan@truni.sk \\
\hline Manara & Alex & Alex.Manara@nbt.nhs.uk \\
\hline Manley & Geoffrey & ManleyG@ucsf.edu \\
\hline Maréchal & Hugues & Hugues.Marechal@chrcitadelle.be \\
\hline Martino & Costanza & costmartino@libero.it \\
\hline Mattern & Julia & Julia.Mattern@med.uni-heidelberg.de \\
\hline McMahon & Catherine & $\begin{array}{l}\text { Catherine.McMahon@thewaltoncentre.nhs. } \\
\text { uk }\end{array}$ \\
\hline Melegh & Béla & bela.melegh@aok.pte.hu \\
\hline Menon & David & dkm13@cam.ac.uk \\
\hline Menovsky & Tomas & tomas.menovsky@uza.be \\
\hline Misset & Benoit & Benoit.Misset@chuliege.be \\
\hline Mulazzi & Davide & davide.mulazzi@policlinico.mi.it \\
\hline Muraleedharan & Visakh & visakh@incf.org \\
\hline Murray & Lynnette & lynnette.murray@monash.edu \\
\hline Nair & Nandesh & nandesh.nair@uza.be \\
\hline Negru & Ancuta & negruancu@gmail.com \\
\hline Nelson & David & david.nelson@karolinska.se \\
\hline Newcombe & Virginia & vfjn2@cam.ac.uk \\
\hline Nieboer & Daan & d.nieboer@erasmusmc.nl \\
\hline Nyirádi & József & nyiradi.jozsef@pte.hu \\
\hline Oresic & Matej & matej.oresic@oru.se \\
\hline
\end{tabular}

(Continued)

\begin{tabular}{|c|c|c|}
\hline Åkerlund & Cecilia & cecilia.ai.akerlund@gmail.com \\
\hline Ortolano & Fabrizio & lupeda@gmail.com \\
\hline Otesile & Olubukola & o.otesile@sheffield.ac.uk \\
\hline Palotie & Aarno & aarno.palotie@helsinki.fi \\
\hline Parizel & Paul M. & paul.parizel@uantwerpen.be \\
\hline Payen & $\begin{array}{l}\text { Jean- } \\
\text { François }\end{array}$ & Jean-Francois.Payen@ujf-grenoble.fr \\
\hline Perera & Natascha & perera@arttic.eu \\
\hline Perlbarg & Vincent & vincent.perlbarg@gmail.com \\
\hline Persona & Paolo & ppersona75@gmail.com \\
\hline Peul & Wilco & W.C.Peul@lumc.nl \\
\hline $\begin{array}{l}\text { Piippo- } \\
\text { Karjalainen }\end{array}$ & Anna & anna.piippo@hus.fi \\
\hline Pirinen & Matti & matti.pirinen@helsinki.fi \\
\hline Ples & Horia & horia.ples@neuromed.ro \\
\hline Polinder & Suzanne & s.polinder@erasmusmc.nl \\
\hline Pomposo & Inigo & inigopomposo@neurocru.com \\
\hline Posti & Jussi P. & jussi.posti@tyks.fi \\
\hline Puybasset & Louis & louis.puybasset@aphp.fr \\
\hline Rădoi & Andreea & aradoi@neurotrauma.net \\
\hline Ragauskas & Arminas & telematics@ktu.lt \\
\hline Raj & Rahul & rahul.raj@hus.fi \\
\hline Rambadagalla & Malinka & malinka.rambadagalla@gmail.com \\
\hline Rehorčíková & Veronika & rehorcikova@gmail.com \\
\hline Rhodes & Jonathan & jrhodes1@staffmail.ed.ac.uk \\
\hline Richardson & Sylvia & sylvia.richardson@mrc-bsu.cam.ac.uk \\
\hline Richter & Sophie & sr773@cam.ac.uk \\
\hline Ripatti & Samuli & samuli.ripatti@helsinki.fi \\
\hline Rocka & Saulius & saulius.rocka@mf.vu.lt \\
\hline Roe & Cecilie & e.c.t.roe@medisin.uio.no \\
\hline Roise & Olav & olav.roise@medisin.uio.no \\
\hline Rosand & Jonathan & jrosand@partners.org \\
\hline Rosenfeld & Jeffrey & J.Rosenfeld@alfred.org.au \\
\hline Rosenlund & Christina & chrisstenrose@gmail.com \\
\hline Rosenthal & Guy & rosenthalg@hadassah.org.il \\
\hline Rossaint & Rolf & RRossaint@ukaachen.de \\
\hline Rossi & Sandra & sandrarossi0@gmail.com \\
\hline Rueckert & Daniel & d.rueckert@imperial.ac.uk \\
\hline Rusnák & Martin & mrusnak@igeh.org \\
\hline Sahuquillo & Juan & sahuquillo@neurotrauma.net \\
\hline Sakowitz & Oliver & oliver.sakowitz@gmail.com \\
\hline Sanchez-Porras & Renan & Renan.Sanchez@kliniken-lb.de \\
\hline Sandor & Janos & sandor.janos@sph.unideb.hu \\
\hline Schäfer & Nadine & Nadine.Schaefer@uni-wh.de \\
\hline Schmidt & Silke & silke.schmidt@uni-greifswald.de \\
\hline Schoechl & Herbert & Herbert.Schoechl@auva.at \\
\hline
\end{tabular}


(Continued)

\begin{tabular}{|c|c|c|}
\hline Åkerlund & Cecilia & cecilia.ai.akerlund@gmail.com \\
\hline Schoonman & Guus & g.schoonman@tsz.nl \\
\hline Schou & Rico Frederik & rico@mymedic.dk \\
\hline Schwendenwein & Elisabeth & $\begin{array}{l}\text { elisabeth.schwendenwein@meduniwien.ac. } \\
\text { at }\end{array}$ \\
\hline Sewalt & Charlie & c.sewalt@erasmusmc.nl \\
\hline Skandsen & Toril & toril.skandsen@ntnu.no \\
\hline Smielewski & Peter & ps10011@cam.ac.uk \\
\hline Sorinola & Abayomi & sorinola_abayomi@hotmail.com \\
\hline Stamatakis & Emmanuel & eas46@cam.ac.uk \\
\hline Stanworth & Simon & simon.stanworth@nhsbt.nhs.uk \\
\hline Kowark & Ana & akowark@ukaachen.de \\
\hline Stevens & Robert & rstevens@jhmi.edu \\
\hline Stewart & William & william.stewart@glasgow.ac.uk \\
\hline Steyerberg & Ewout W. & e.steyerberg@erasmusmc.nl \\
\hline Stocchetti & Nino & stocchet@policlinico.mi.it \\
\hline Sundström & Nina & Nina.Sundstrom@vll.se \\
\hline Synnot & Anneliese & anneliese.synnot@monash.edu \\
\hline Takala & Riikka & riikka.takala@tyks.fi \\
\hline Tamás & Viktória & tamas.viktoria@pte.hu \\
\hline Tamosuitis & Tomas & tomas.tamosuitis@kaunoklinikos.lt \\
\hline Taylor & Mark Steven & marktrnava@gmail.com \\
\hline Te Ao & Braden & braden.teao@aut.ac.nz \\
\hline Tenovuo & Olli & olli.tenovuo@tyks.fi \\
\hline Theadom & Alice & alice.theadom@aut.ac.nz \\
\hline Thomas & Matt & Matt.Thomas@nbt.nhs.uk \\
\hline Tibboel & Dick & d.tibboel@erasmusmc.nl \\
\hline Timmers & Marjolein & mtimmers@hotmail.com \\
\hline Tolias & Christos & christos.tolias@nhs.net \\
\hline Trapani & Tony & tony.trapani@monash.edu \\
\hline Tudora & $\begin{array}{l}\text { Cristina } \\
\text { Maria }\end{array}$ & cristina.tudora@neuromed.ro \\
\hline Vajkoczy & Peter & Peter.Vajkoczy@charite.de \\
\hline Valeinis & Egils & Egils.Valeinis@latnet.Iv \\
\hline Vallance & Shirley & S.Vallance@alfred.org.au \\
\hline Vámos & Zoltán & azozoka@gmail.com \\
\hline van der Naalt & Joukje & j.van.der.naalt@umcg.nl \\
\hline Van der Steen & Gregory & gregory@webstone.be \\
\hline van Dijck & Jeroen T.J.M. & j.t.j.m.van_dijck@lumc.nl \\
\hline van Essen & Thomas A. & T.A.van_Essen@lumc.nl \\
\hline Van Hecke & Wim & wim.vanhecke@icometrix.com \\
\hline van Heugten & Caroline & $\begin{array}{l}\text { Caroline.vanheugten@maastrichtuniversity. } \\
\mathrm{nl}\end{array}$ \\
\hline Van Praag & Dominique & dominique.vanpraag@uza.be \\
\hline van Wijk & Roel & roel-van-wijk@ziggo.nl \\
\hline Vande Vyvere & Thijs & thijs.vandevyvere@icometrix.com \\
\hline
\end{tabular}

(Continued)

\begin{tabular}{|c|c|c|}
\hline Åkerlund & Cecilia & cecilia.ai.akerlund@gmail.com \\
\hline Vargiolu & Alessia & neurorianimazione@hsgerardo.org \\
\hline Vega & Emmanuel & emmanuel.vega@chru-lille.fr \\
\hline Velt & Kimberley & k.velt@erasmusmc.nl \\
\hline Verheyden & Jan & jan.verheyden@icometrix.com \\
\hline Vespa & Paul M. & PVespa@mednet.ucla.edu \\
\hline Vik & Anne & anne.vik@ntnu.no \\
\hline Vilcinis & Rimantas & rimantas.vilcinis@kaunoklinikos.lt \\
\hline Volovici & Victor & v.volovici@erasmusmc.nl \\
\hline von Steinbüchel & Nicole & nvsteinbuechel@med.uni-goettingen.de \\
\hline Voormolen & Daphne & d.voormolen@erasmusmc.nl \\
\hline Vulekovic & Petar & pvulekovic@gmail.com \\
\hline Wang & Kevin K.W. & kawangwang17@gmail.com \\
\hline Wiegers & Eveline & e.wiegers@erasmusmc.nl \\
\hline Williams & Guy & gbw1000@wbic.cam.ac.uk \\
\hline Wilson & Lindsay & I.wilson@stir.ac.uk \\
\hline Winzeck & Stefan & sw742@cam.ac.uk \\
\hline Wolf & Stefan & stefan.wolf@charite.de \\
\hline Yang & Zhihui & zhihuiyang@ufl.edu \\
\hline Ylén & Peter & peter.ylen@vtt.fi \\
\hline Younsi & Alexander & alexander.younsi@med.uni-heidelberg.de \\
\hline Zeiler & Frederick A. & umzeiler@myumanitoba.ca \\
\hline Ziverte & Agate & agate.ziverte@inbox.lv \\
\hline Zoerle & Tommaso & tommaso.zoerle@policlinico.mi.it \\
\hline
\end{tabular}

\section{Authors' contributions}

$M T, J D, R W, V L, E V, A M, D M, G C, N S$, EK participated in the conceptualization of the manuscript. MT and JD contributed equally, collected the data with VL and drafted the manuscript and the supplementary files. MT, JD, EV and RW analyzed the data. MT, JD, RW, VL, EV, AM, DM, GC, NS, EK had a role in data interpretation and provided feedback on the manuscript. EK supervised the project. MT, JD, RW, VL, EV, AM, DM, GC, NS, EK approved the submitted final version of the manuscript.

\section{Funding}

CENTER-TBI was supported by the European Union 7th Framework program (EC grant 602150). Additional funding was obtained from the Hannelore Kohl Stiftung (Germany), from OneMind (USA) and from Integra LifeSciences Corporation (USA). David K. Menon was supported by a Senior Investigator Award from the National Institute for Health Research (UK). The funders had no role in study design, data collection and analysis, decision to publish, or preparation of the manuscript.

\section{Availability of data and materials}

There are legal constraints that prohibit us from making all data publicly available. Data could be identifiable because the limited number of centres per country that were included in this study. Readers may contact Dr. Erwin J. O. Kompanje (erwinkompanje@me.com) for reasonable requests for the data. 


\section{Ethics approval and consent to participate}

All IRBs approved the CENTER-TBI research protocol and the assessment of IRB data. A complete list can be found on https://www.center-tbi.eu/project/ ethical-approval.

\section{Consent for publication}

Not applicable.

\section{Competing interests}

The authors declare that they have no competing interests.

\section{Author details}

'Department of Intensive Care, Erasmus MC - University Medical Centre Rotterdam, P.O. Box 2040, 3000 CA Rotterdam, the Netherlands. ${ }^{2}$ Department of Neurosurgery, University Neurosurgical Center Holland, LUMC, HMC \& Haga Teaching Hospital, Leiden, The Hague, The Netherlands. ${ }^{3}$ ICON plc, South County Business Park Leopardstown, Dublin 18, Ireland. ${ }^{4}$ Department of Public Health, Erasmus MC - University Medical Centre Rotterdam, Rotterdam, the Netherlands. ${ }^{5}$ Department of Neurosurgery, Antwerp University Hospital, Edegem, Belgium. ${ }^{6}$ University of Antwerp, Antwerp, Belgium. ${ }^{7}$ Department of Anaesthesia, University of Cambridge, Cambridge, UK. ${ }^{8}$ School of Medicine and Surgery, University of Milan-Bicocca, Milan, Italy. ${ }^{9}$ San Gerardo Hospital, ASST-Monza, Monza, Italy. ${ }^{10}$ Department of Physiopathology and Transplantation, Milan University, Milan, Italy. ${ }^{11}$ Neuro ICU Fondazione IRCCS Cà Granda Ospedale Maggiore Policlinico Milano, Milan, Italy. ${ }^{12}$ Department of Medical Ethics and Philosophy of Medicine, Erasmus MC - University Medical Center Rotterdam, Rotterdam, the Netherlands.

Received: 9 November 2019 Accepted: 1 May 2020

Published online: 12 May 2020

\section{References}

1. Steering Commitee on Bioethics, Council of Europe. Guide for Research Ethics Committee Members, revised version 3 December 2010. https://www. coe.int/t/dg3/healthbioethic/activities/02_biomedical_research_en/Guide/ Guide_EN.pdf. Accessed 3 Sept 2019.

2. Emanuel E, Crouch R, Lie R, et al. The Oxford textbook of clinical research ethics. Oxford: Oxford University Press, Reprint edition; 2011

3. World Health Organization. Standards and operational guidance for ethics review of health-related research with human participants. 2011. https:// apps.who.int/iris/bitstream/handle/10665/44783/9789241502948_eng.pdf; jsessionid $=0$ CC3C3EA5BABF39889211B2E3B4AA76B? sequence $=1$. Accessed 3 Sept 2019

4. Innovative Medicine Innitiative, IMI mission and objectives. https://www.imi. europa.eu/about-imi/mission-objectives. Accessed August 3, 2019.

5. European Commission, Horizon 2020 - The Framework Programme for Research and Innovation. https://eur-lex.europa.eu/legal-content/EN/TXT/ PDF/?uri=CELEX:52011DC0808\&from=EN. Published 2011. Accessed September 3, 2019.

6. Tridente A, Holloway P, Hutton P, et al. Methodological challenges in European ethics approvals for a genetic epidemiology study in critically ill patients: the GenOSept experience. BMC Med Ethics. 2019;20:30.

7. Urushihara H, Parmenter L, Tashiro S, et al. Bridge the gap: the need for harmonized regulatory and ethical standards for postmarketing observational studies. Pharmacoepidemiol Drug Saf. 2017;26:1299-306.

8. Aledort L. Harmonization of clinical trial guidelines for assessing the risk of inhibitor development in hemophilia a treatment. J Thromb Haemost. 2011; 9(3):423-7.

9. Oliver DJ. Harmonisation of research outcomes for meaningful translation to practice: the role of Core outcome sets and the CROWN initiative. Aust N Z J Obs Gynaecol. 2018;58:15-6.

10. Bowles K, Potashnik S, Ratcliffe $\mathrm{S}$, et al. Conducting research using the electronic health record across multi-hospital systems: semantic harmonization implications for administrators. J Nurs Adm. 2013:43:355-60.

11. Maas A, Menon D, Steyerberg E, et al. Collaborative European NeuroTrauma effectiveness research in traumatic brain injury (CENTER-TBI): a prospective longitudinal observational study. Neurosurgery. 2015;76:67-80.

12. Steyerberg E, Wiegers $\mathrm{E}$, Sewalt $\mathrm{C}$, et al. Case-mix, care pathways, and outcomes in patients with traumatic brain injury in CENTER-TBI: a European prospective, multicentre, longitudinal, cohort study. Lancet Neurol. 2019;18: 923-34.

13. Maas A, Menon D, Adelson P, et al. Traumatic brain injury: integrated approaches to improve prevention, clinical care, and research. Lancet Neurol. 2017;16:987-1048.

14. Cnossen $\mathrm{M}$, Polinder $\mathrm{S}$, Lingsma $\mathrm{H}$, et al. Variation in structure and process of care in traumatic brain injury: provider profiles of European Neurotrauma centers participating in the CENTER-TBI study. PLoS One. 2016;11(8): e0161367.

15. ICON plc. http://www.iconplc.com. Accessed August 8, 2019.

16. United Nations, Standard country or area codes for statistical use (M49). https://unstats.un.org/unsd/methodology/m49/. Published 1999. Accessed September 5, 2019.

17. Mascette A, Bernard G, Dimichele D, et al. Are central institutional review boards the solution? The National Heart, Lung, and Blood Institute working Group's report on optimizing the IRB process. Acad Med. 2012;87:1710-4.

18. Adams P, Kaewkungwal J, Limphattharacharoen C, et al. Is your ethics committee efficient? Using "RB Metrics" as a self-assessment tool for continuous improvement at the Faculty of Tropical Medicine, Mahidol University, Thailand. PLoS ONE. 2014;9(11):e113356.

19. Thiese M. Observational and interventional study design types; an overview. Biochem Med. 2014:24(2):199-210.

20. Ravina B, Deuel L, Siderowf A, et al. Local institutional review board (IRB) review of a multicenter trial: local costs without local context. Ann Neurol. 2010;67(2):258-60

21. European Union. Consolidated version of the treaty on the functioning of the european union. Official Journal of the European Union C 326/47. 2012. https://eur-lex.europa.eu/legal-content/EN/ALL/?uri=OJ\%3AC\%3A2012\%3 A326\%3ATOC. Accessed 9 Sept 2019

22. Henshall C, Mardhani-Bayne L, Fronsdal KB, et al. Interactions between health technology assessment, coverage, and regulatory processes: emerging issues, goals, and opportunities. Int J Technol Assess Heal Care. 2011:27(3):253-60

23. European Union. Regulation (EU) 2016/679 of the European Parliament and of the Council of 27 April 2016 on the protection of natural persons with regard to the processing of personal data and on the free movement of such data, and repealing Directive 95/46/EC (General data Protection Regulation). Official Journal of the European Union L 119/1, version 4 May 2016. http://data.europa.eu/eli/reg/2016/679/oj. Accessed 9 Sept 2019.

24. European Commission. The European Union. What it is and what it does. Luxembourg: Publications Office of the European Union 2018. doi:https:// doi.org/10.2775/665897.

25. Timmers $M$, Van Veen E-B, Maas A, et al. Will the Eu data protection regulation 2016/679 inhibit critical care research? Med Law Rev. 2019;27:59-78.

\section{Publisher's Note}

Springer Nature remains neutral with regard to jurisdictional claims in published maps and institutional affiliations.

Ready to submit your research? Choose BMC and benefit from:

- fast, convenient online submission

- thorough peer review by experienced researchers in your field

- rapid publication on acceptance

- support for research data, including large and complex data types

- gold Open Access which fosters wider collaboration and increased citations

- maximum visibility for your research: over $100 \mathrm{M}$ website views per year

At BMC, research is always in progress.

Learn more biomedcentral.com/submissions 